\title{
Aberrant methylation of TRIM58 in hepatocellular carcinoma and its potential clinical implication
}

\author{
XUEPING QIU*, YIFANG HUANG* ${ }^{*}$ YE ZHOU and FANG ZHENG \\ Center for Gene Diagnosis, Zhongnan Hospital of Wuhan University, Wuhan, Hubei 430071, P.R. China
}

Received February 1, 2016; Accepted March 15, 2016

DOI: $10.3892 /$ or.2016.4871

\begin{abstract}
TRIM58 (tripartite motif containing 58) has been reported as a novel methylated gene in hepatocellular carcinoma (HCC) by methylation microarrays. However, its associations with mRNA expression and clinicopathological characteristics have not been evaluated. In this study, we explored the potential clinical implications of TRIM58 methylation in HCC. We analyzed the methylation level of TRIM58 in $181 \mathrm{HCC}$ tissues, 172 matched adjacent non-tumor tissues and 13 normal liver tissues using methylation-sensitive restriction enzyme based quantitative PCR and bisulfite genomic sequencing. Further, the mRNA expression level of TRIM58 was measured in 46 paired HCC and adjacent non-tumor tissues by quantitative real-time PCR. Moreover, the relationship between TRIM58 methylation and mRNA expression, the clinicopathological features, as well as prognostic value were evaluated. The results showed that TRIM58 methylation was significantly higher in HCC tissues compared with adjacent non-tumor tissues and normal liver tissues (both $\mathrm{p}<0.0001$ ). Using $10 \%$ as the cut-off value, hypermethylation of TRIM58 was specific in HCC tissues $(28.18 \%, 51 / 181)$, with a tendency to correlate with unfavorable disease-free survival $(\mathrm{p}=0.047)$. Moreover, TRIM58 expression was significantly decreased in HCC tissues compared with adjacent non-tumor tissues $(\mathrm{p}<0.0001)$, and showed a negative association with DNA methylation $\left(\mathrm{p}=0.015, \mathrm{r}_{\mathrm{s}}=-0.260\right)$. Our data indicate that TRIM58 methylation is a common event in $\mathrm{HCC}$ and may contribute to downregulation of its mRNA expression. Furthermore, hypermethylation of TRIM58 tends to be associated with worse DFS after hepatectomy. However, the potential clinical application of TRIM58 need to be further investigated.
\end{abstract}

Correspondence to: Professor Fang Zheng, Center for Gene Diagnosis, Zhongnan Hospital of Wuhan University, 169 Donghu Road, Wuchang, Wuhan, Hubei 430071, P.R. China

E-mail: zhengfang@whu.edu.cn

${ }^{*}$ Contributed equally

Key words: TRIM58, DNA methylation, mRNA expression, hepatocellular carcinoma

\section{Introduction}

Hepatocellular carcinoma (HCC) is one of the most common and aggressive malignancies worldwide (1). The major risk factors contributing to $\mathrm{HCC}$ include infection with hepatitis $\mathrm{B}$ or $\mathrm{C}$ virus, non-alcoholic steatohepatitis disease, aflatoxin $\mathrm{B}$ exposure and chronic alcoholism $(2,3)$. It is widely accepted that hepatocarcinogenesis is a multi-step process of serial genetic and epigenetic abnormalities (4-6). DNA methylation, the best-characterized epigenetic mechanism, has been reported as one of the pivotal alterations in HCC development and progression $(7,8)$. Global genomic hypomethylation and gene specific hypermethylation are two main patterns of aberrant DNA methylation, and these alterations coexist in HCC. Growing evidence showed that aberrant methylation presents potential clinical applications for HCC detection, diagnosis and prognosis $(9,10)$. Therefore, evaluating the status of DNA methylation could aid in identifying molecular biomarkers that may have potential applications in clinical practice for HCC.

TRIM58 (tripartite motif containing 58) is a member of the tripartite motif-containing family. The TRIM proteins frequently possess E3 ubiquitin ligase activities and participate in a broad range of physiological processes and disease, including innate immunity, development process, genetic diseases, and cancer (11). It has been reported that TRIM58 regulated terminal erythroid cell cycles and enucleation (12). However, the association between TRIM58 and HCC has not been well documented. Recently, hypermethylation of TRIM58 has been detected in hepatocellular carcinoma using methylation microarrays, and further validated in 10 paired tumor and adjacent liver tissues using combined bisulfite restriction analysis (COBRA) (13). Additionally, Tao et al also found that TRIM58 mRNA expression was upregulated after 5-aza-dc treatment in HCC cell lines, which indicated that TRIM58 methylation was tightly associated with its downregulation in HCC cell lines. However, this observation based on cell lines was not validated in clinical specimens. Therefore, it is necessary to revaluate the methylation and expression of TRIM58 in a larger cohort of clinical samples and explore its potential clinical implication.

In the present study we measured the methylation level and mRNA expression of TRIM58 in HCC and adjacent non-tumor tissues by MSRE-qPCR and quantitative real-time PCR. Correlations between the methylation and mRNA expression, and clinicopathological features were evaluated. 


\section{Materials and methods}

Tissue samples. A total of 181 HCC tissues and 172 adjacent non-tumor tissues (including 172 paired samples) were obtained from HCC patients who underwent surgical resections from May 2011 to November 2014 at Zhongnan Hospital of Wuhan University and Hubei Cancer Hospital. In addition, 13 normal tissues from patients with hepatic hemangiomas were included as control. Among these samples, 76 pairs of HCC tissues and matched adjacent non-tumor tissues were fresh-frozen tissues that were stored at $-80^{\circ} \mathrm{C}$ immediately after surgery, whereas the others were formalin-fixed paraffinembedded (FFPE) samples.

All patients were diagnosed by ultrasonography or computed tomography, and confirmed by histology of liver biopsy. None of the patients had an additional history of a solid organ tumor or preoperative therapy. This study was approved by the Medical Ethical Committee of Zhongnan Hospital of Wuhan University and followed the tenets of the Declaration of Helsinki and its later amendments. Informed consent was obtained from all individuals before the study carried out. The demographic clinicopathological information was obtained from the electronic medical records of patients, which are shown in Table I.

Bisulfite genomic sequencing (BGS) and methylationsensitive restriction enzyme digestion and quantitative PCR $(M S R E-q P C R)$. DNA from FFPE samples and fresh-frozen tissues was isolated using QIAamp DNA FFPE Tissue kit (Qiagen) and standard phenol/chloroform extraction, respectively. DNA was quantified by the NanoDrop-2000c (Thermo Scientific, Rockford, IL, USA) and stored at $-20^{\circ} \mathrm{C}$ until use. Bisulphite conversion and bisulfite genomic sequencing (BGS) were performed as previously described (14). The methylation level of TRIM58 was calculated as follows: $100 \% \mathrm{x}$ methylated CG / (methylated CG + unmethylated CG). MSRE-qPCR was performed to detect the methylation level of TRIM58 as our previous study (14). ACTB without methylation-sensitive restriction enzyme recognition site was used as an internal control. The methylation level of TRIM58 was measured by $100 \% \times 2^{-\Delta C q}$ (undigested - digested) after normalization to the ACTB (15). The primers used for BGS and MSRE-qPCR were listed in Table II.

RNA isolation and gene expression analysis. Total RNA was isolated from fresh-frozen tissues using TRIzol Reagent (Life Technologies, Gaithersburg, MD, USA) according to the manufacturer's instructions. The cDNA was synthesized from $1 \mu \mathrm{g}$ RNA by PrimeScript ${ }^{\mathrm{TM}}$ RT reagent kit with gDNA Eraser (Takara, Dalian, China). The expression level of TRIM58 was performed in duplicate by quantitative PCR using Bio-Rad CFX96 ${ }^{\mathrm{TM}}$ real-time PCR detection system (Bio-Rad, Hercules, CA, USA) in accordance with the manufacturer's protocol. Each assay was carried out in a total volume of $20 \mu \mathrm{l}$, including 1X SYBR ${ }^{\circledR}$ Premix Ex Taq ${ }^{\mathrm{TM}}$ GC (Takara), $0.5 \mu \mathrm{M}$ of forward and reverse primers (Table II), $2 \mu \mathrm{l}$ cDNA template. Non-template control was used as negative control, and melting curve analysis was employed to verify the specificity of PCR product. All reactions were normalized to ACTB as an internal control, and the relative expression level of TRIM58 was determined by using the comparative $\mathrm{Cq}$ method $\left(2^{-\mathrm{ACq}}\right)$.
Table I. Clinicopathological characteristics of patients with HCC.

\begin{tabular}{|c|c|}
\hline Characteristics & No. $(\%)$ \\
\hline Total case number & 181 \\
\hline \multicolumn{2}{|l|}{ Gender } \\
\hline Male & $154(85.08 \%)$ \\
\hline Female & $27(14.92 \%)$ \\
\hline \multicolumn{2}{|l|}{ Age at diagnosis } \\
\hline$<55$ years & $96(53.04 \%)$ \\
\hline$\geq 55$ years & $83(45.86 \%)$ \\
\hline Missing & $2(1.10 \%)$ \\
\hline Mean \pm SD & $52.82 \pm 11.06$ \\
\hline Median (interquartile range) & $54(45-61)$ \\
\hline \multicolumn{2}{|l|}{ Etiology } \\
\hline $\mathrm{HBV}(+) \mathrm{HCV}(-)$ & $166(91.71 \%)$ \\
\hline HBV (-) HCV (+) & $2(1.10 \%)$ \\
\hline $\mathrm{HBV}(+) \mathrm{HCV}(+)$ & $6(3.31 \%)$ \\
\hline HBV (-) HCV (-) & $2(1.10 \%)$ \\
\hline Missing & $5(2.76 \%)$ \\
\hline \multicolumn{2}{|l|}{ Child-Pugh class } \\
\hline A & $167(92.27 \%)$ \\
\hline $\mathrm{B}$ & $9(4.97 \%)$ \\
\hline $\mathrm{C}$ & $0(0.0 \%)$ \\
\hline Missing & $5(2.76 \%)$ \\
\hline \multicolumn{2}{|l|}{ Serum AFP $(\mathrm{ng} / \mathrm{ml})$} \\
\hline Negative $(<20)$ & $59(32.60 \%)$ \\
\hline Positive $(\geq 20)$ & $114(62.98 \%)$ \\
\hline Missing & $8(4.42 \%)$ \\
\hline Negative $(<200)$ & $96(53.04 \%)$ \\
\hline Positive $(\geq 200)$ & $77(42.54 \%)$ \\
\hline \multicolumn{2}{|l|}{ Cirrhosis } \\
\hline Negative & $36(19.89 \%)$ \\
\hline Positive & $139(76.80 \%)$ \\
\hline Missing & $6(3.31 \%)$ \\
\hline \multicolumn{2}{|l|}{ Size of tumor $(\mathrm{cm})$} \\
\hline$<5.0$ & $62(34.25 \%)$ \\
\hline$\geq 5.0$ & $114(62.98 \%)$ \\
\hline Missing & $5(2.76 \%)$ \\
\hline \multicolumn{2}{|l|}{ Number of tumor(s) } \\
\hline Single & $142(78.45 \%)$ \\
\hline Multiple & $34(18.78 \%)$ \\
\hline Missing & $5(2.76 \%)$ \\
\hline \multicolumn{2}{|l|}{ Tumor embolus } \\
\hline Negative & $140(77.35 \%)$ \\
\hline Positive & $35(19.34 \%)$ \\
\hline Missing & $6(3.31 \%)$ \\
\hline \multicolumn{2}{|l|}{ Differentiation (Edmondson) } \\
\hline I & $16(8.84 \%)$ \\
\hline II & $109(60.22 \%)$ \\
\hline III & $50(27.62 \%)$ \\
\hline IV & $2(1.10 \%)$ \\
\hline Missing & $4(2.21 \%)$ \\
\hline \multicolumn{2}{|l|}{ TNM stage } \\
\hline $\mathrm{I}$ & $105(58.01 \%)$ \\
\hline II & $17(9.39 \%)$ \\
\hline III & $54(29.83 \%)$ \\
\hline IV & $0(0.0 \%)$ \\
\hline Missing & $5(2.76 \%)$ \\
\hline
\end{tabular}

$\mathrm{HBV}$, hepatitis B virus; $\mathrm{HCV}$, hepatitis $\mathrm{C}$ virus; $\mathrm{AFP}$, alpha-fetoprotein; TNM, tumor-node-metastasis. 

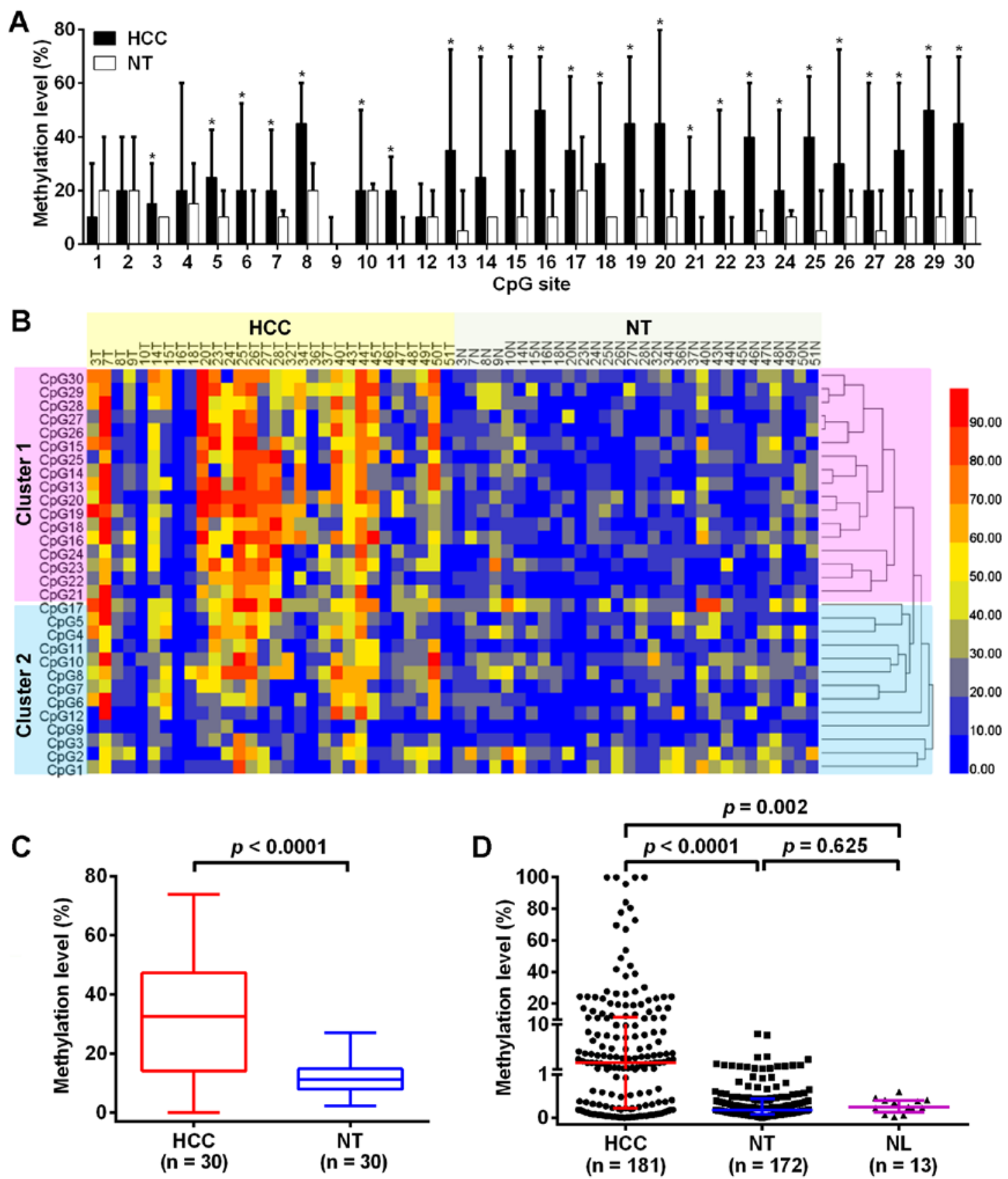

Figure 1. Methylation analysis of TRIM58 CpG islands (CGI) in liver tissues. (A) Methylation levels of each CpG site in TRIM58 CGI region were compared between HCC tissues and adjacent non-tumor tissues. "p $<0.05$, Wilcoxon matched pairs test. (B) Heatmap showed unsupervised cluster of TRIM58 methylation at each CpG sites between HCC tissues and adjacent non-tumor tissues. Blue corresponded to low methylation and red to high methylation. (C) Boxplot of TRIM58 methylation in 30 paired HCC and adjacent non-tumor tissues using BGS. The p-value was calculated by Wilcoxon matched pairs test. (D) TRIM58 methylation was determined in $181 \mathrm{HCC}$ tissues, 172 adjacent non-tumor tissues and 13 normal liver tissues by MSRE-qPCR. The bars represented the median value with interquartile range and p-value was calculated by Mann-Whitney U test. HCC, hepatocellular carcinoma tissues; NT, adjacent non-tumor tissues; $\mathrm{NL}$, normal liver tissues.

Statistical analysis. The comparison of TRIM58 methylation level between various subgroups was conducted using Wilcoxon matched pairs test or Mann-Whitney U test where necessary. Paired-samples T-test was used to compare the expression of TRIM58 ( $\log 10$ transformation) between HCC tissues and adjacent non-tumor tissues. The correlation between two continuous variables was evaluated by Spearman's rank correlation. For survival analysis, Kaplan-Meier method with log-rank test and COX regression model were performed to elucidate the prognostic significance of TRIM58 methylation.
The statistical analyses were conducted with the SPSS 16.0 and GraphPad Prism 6.0, all p-values were two-sided and $\mathrm{p}<0.05$ was considered statistically significant.

\section{Results}

Hypermethylation of TRIM58 in HCC. To investigate the methylation status of TRIM58 in HCC, we firstly evaluated the methylation level in 30 paired HCC tissues and adjacent non-tumor tissues using BGS. A total of $30 \mathrm{CpG}$ sites were 


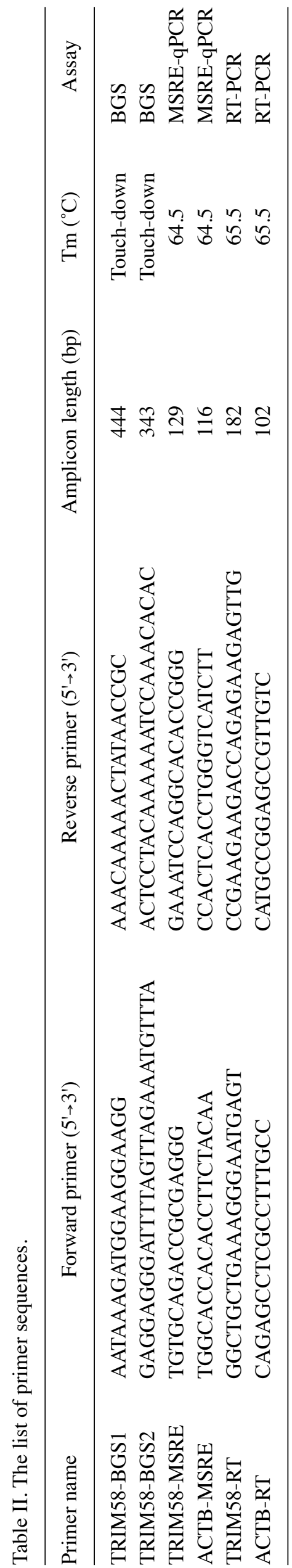

detected and the methylation levels were varied in different CpG sites (Fig. 1A). The methylation level of TRIM58 in HCC tissues were significantly higher than that in paired adjacent non-tumor tissues at each $\mathrm{CpG}$ site $(\mathrm{p}<0.05)$, excepting the CpG sites at 1, 2, 4, 9 and 12 ( $\mathrm{p}>0.05)$. A hierarchical clustering analysis was performed according to the methylation level of each $\mathrm{CpG}$ site in 60 liver tissues, and the $30 \mathrm{CpG}$ sites were divided into two distinct subclasses, cluster 1 and cluster 2 (Fig. 1B). The methylation differences between HCC and adjacent non-tumor tissues at $\mathrm{CpG}$ sites in cluster 1 were more obvious than in cluster 2 . The overall methylation of TRIM58 in HCC tissues was also significantly higher than that in matched adjacent non-tumor tissues [32.50\% (14.17\%$47.17 \%$ ) vs. $11.17 \%$ (7.92\%-14.75\%), p<0.0001, Fig. 1C].

To further explore the association between TRIM58 methylation and clinicopathological characteristics, 181 HCC tissues, 172 matched adjacent non-tumor tissues and 13 normal liver tissues were used to verify the methylation level of TRIM58 in the region between CpG site 13 and 30 (cluster 1) using MSRE-qPCR. Representative results of BGS and MSRE-qPCR are displayed in Fig. 2. The result from MSRE-qPCR showed that the methylation level of TRIM58 in HCC tissues was significantly higher than that in adjacent non-tumor tissues and normal liver tissues $(\mathrm{p}<0.0001$ and $\mathrm{p}=0.002$, respectively, Fig. 1D). However, there was no significant difference in methylation level of TRIM58 between the adjacent non-tumor tissues and normal liver tissues $(\mathrm{p}=0.625$, Fig. 1D). With a $10 \%$ hypermethylation level threshold established $(16,17), 28.18 \%(51 / 181)$ of HCC specimens showed increased hypermethylation, as opposed to $0 \%$ in the adjacent non-tumor tissues and normal liver tissues. Overall, both data from BGS and MSRE-qPCR indicated a significant increase in the methylation level of TRIM58 in HCC tissues compared with the adjacent non-tumor tissues.

Correlations between TRIM58 methylation and clinicopathological features. The correlation between TRIM58 methylation and clinicopathological features of 181 HCC samples are summarized in Table III. The methylation level of TRIM58 was increased in patients with serum AFP $\geq 200 \mathrm{ng} / \mathrm{ml}(\mathrm{p}=0.015)$, tumor embolus $(\mathrm{p}=0.0026)$ and advanced tumor-node-metastasis (TNM) stage $(\mathrm{p}=0.046)$, respectively. No other correlations were observed between the methylation of TRIM58 and clinicopathological parameters, such as patient gender, age, cirrhosis, tumor number and size, as well as tumor differentiation $(\mathrm{p}>0.05)$. Spearman's rank order correlation coefficient was calculated to further analyze associations between TRIM58 methylation and AFP, tumor number and size as continuous variables. The results indicated that the methylation of TRIM58 had a positive significant correlations with AFP level $\left(\mathrm{p}=0.037, \mathrm{r}_{\mathrm{s}}=0.159\right)$. However, no correlations between TRIM58 methylation and tumor number and size were observed.

TRIM58 hypermethylation tend to be associated with worse disease-free survival. The follow-up began with the date of HCC resection and ended with the date of death or the last clinical review before June 30, 2015. Survival analysis was finally conducted in $67 \mathrm{HCC}$ patients with the follow-up time more than 3 months. The median follow-up time was 10 months 

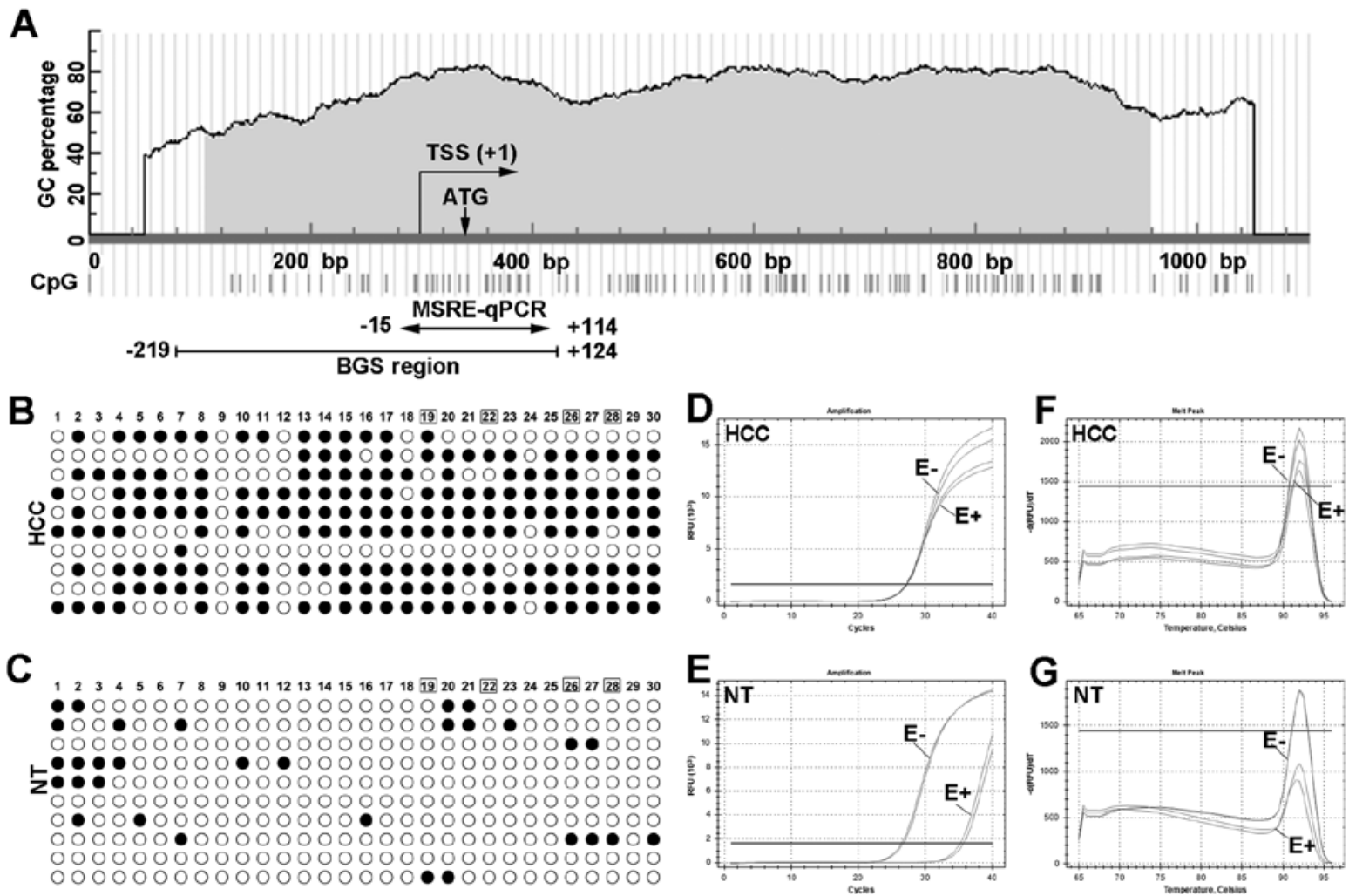

Figure 2. Representative results of TRIM58 methylation in HCC and adjacent non-tumor liver tissues using BGS and MSRE-qPCR. (A) Schematic structure of TRIM58 CGI, including the transcript start site (TSS), translation initiation site (ATG), BGS and MSRE-qPCR region. The position of BGS and MSREqPCR are marked with arrows, and the number indicates the relative position to TSS (TSS was considered as +1). (B and C) TRIM58 methylation in HCC and adjacent non-tumor liver tissues using BGS. Each row represents the sequencing result from one clone, and the number represents the position of CpG sites in target region (number 1 indicate the first CpG site in the target region). Open and closed circles represent methylated and unmethylated CG dinucleotides, respectively. The CpG sites within recognition sites of MSRE are marked in box (19, 22, 26 and 28). MSRE-qPCR results for the same sample of BGS are displayed as amplification curve (D and E) and melt curve (F and G). The line marked as E+ and E- indicate digested sample and undigested sample for TRIM58, respectively. HCC, hepatocellular carcinoma tissues; NT, adjacent non-tumor tissues.

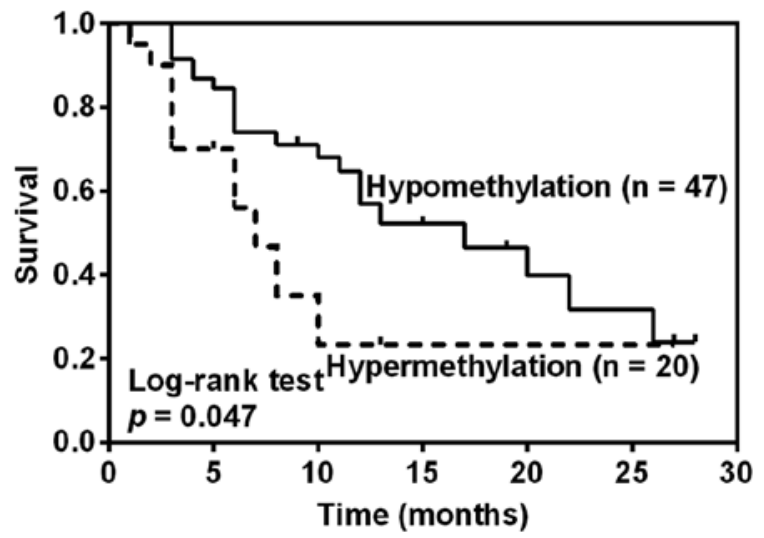

Figure 3. Kaplan-Meier curves for disease-free survival (DFS) rate of patients with HCC according to the methylation level of TRIM58 at diagnosis ( $\mathrm{n}=67)$. The solid line represented patients with methylation $\geq 10 \%$; the dashed line represented the patients with methylation $<10 \%$.

(range, 3-33 months). The median disease-free survival time (DFS, defined as survival without any clinical evidence of recurrence or metastasis) was 12.33 months and 32 patients developed recurrence or metastasis. The overall survival analysis was not performed due to the death of only two patients. To explore the association between TRIM58 methylation and
DFS, patients were divided into groups of hypomethylation or hypermethylation according to the threshold of TRIM58 methylation level established (10\%). Kaplan-Meier analysis revealed that HCC patients with TRIM58 hypermethylation showed a significantly shorter median DFS after hepatectomy than those with TRIM58 hypomethylation (7 months vs. 17 months, $\mathrm{p}=0.047$, Fig. 3). Furthermore, COX regression model showed that larger tumor size $(\mathrm{p}=0.0002, \mathrm{HR}=1.113$, 95\% CI: 1.059-1.205) and advanced differentiation $(\mathrm{p}=0.013$, HR=2.448, 95\% CI: 1.206-4.972), but not TRIM58 hypermethylation ( $>0.05$ ), were independent prognostic predictors for unfavorable DFS (data not shown).

TRIM58 mRNA expression was downregulated in HCC and inversely associated with its methylation. To further study the relationship between TRIM58 methylation and mRNA expression, we measured the mRNA expression of TRIM58 in 43 of the 172 paired HCC and adjacent non-tumor tissues by quantitative real-time PCR. Considering 0.5 as the cut-off value, TRIM58 mRNA expression was downregulated in about 79\% (34/43) of HCC tissues (Fig. 4A) and the difference was statistically significant $(\mathrm{p}<0.0001$, Fig. 4B). Moreover, TRIM58 methylation was significantly higher in HCC specimens ( $p<0.0001$, Fig. 4C) and had an inverse correlation with its mRNA expression, despite a lower correlation coefficient $(\mathrm{p}=0.015, \mathrm{rs}=-0.260)$. 
Table III. Associations between TRIM58 methylation and clinicopathological parameters in 181 HCC patients.

\begin{tabular}{|c|c|c|c|}
\hline Characteristics & No. $(\%)$ & Methylation level (\%) ${ }^{\mathrm{a}}$ & $\mathrm{p}$-value ${ }^{\mathrm{b}}$ \\
\hline Gender & & & 0.992 \\
\hline Male & $154(85.08 \%)$ & $2.54(0.21-12.37)$ & \\
\hline Female & $27(14.92 \%)$ & $1.38(0.32-7.57)$ & \\
\hline Age at diagnosis & & & 0.073 \\
\hline$<55$ years & $96(53.04 \%)$ & $3.00(0.38-18.82)$ & \\
\hline$\geq 55$ years & $83(45.86 \%)$ & $1.04(0.14-10.86)$ & \\
\hline Missing & $2(1.10 \%)$ & & \\
\hline Serum AFP (ng/ml) & & & 0.397 \\
\hline Negative $(<20)$ & $59(32.60 \%)$ & $2.14(0.19-7.57)$ & \\
\hline Positive ( $\geq 20)$ & $114(62.98 \%)$ & $2.73(0.22-15.6)$ & \\
\hline Missing & $8(4.42 \%)$ & & \\
\hline Serum AFP (ng/ml) & & & 0.015 \\
\hline Negative $(<200)$ & $96(53.04 \%)$ & $17.98(0.84-58.83)$ & \\
\hline Positive $(\geq 200)$ & $77(42.54 \%)$ & $25.12(2.21-56.08)$ & \\
\hline Missing & $8(4.42 \%)$ & & \\
\hline Cirrhosis & & & 0.444 \\
\hline Negative & $36(19.89 \%)$ & $0.99(0.08-19.59)$ & \\
\hline Positive & $139(76.80 \%)$ & $2.83(0.25-10.86)$ & \\
\hline Missing & $6(3.31 \%)$ & & \\
\hline Size of tumor $(\mathrm{cm})$ & & & 0.229 \\
\hline$<5.0$ & $62(34.25 \%)$ & $1.33(0.24-7.00)$ & \\
\hline$\geq 5.0$ & $114(62.98 \%)$ & $3.22(0.19-17.00)$ & \\
\hline Missing & $5(2.76 \%)$ & & \\
\hline Number of tumor(s) & & & 0.326 \\
\hline Single & $142(78.45 \%)$ & $2.20(0.21-10.92)$ & \\
\hline Multiple & $34(18.78 \%)$ & $3.4(0.36-11.98)$ & \\
\hline Missing & $5(2.76 \%)$ & & \\
\hline Tumor embolus & & & 0.026 \\
\hline Negative & $140(77.35 \%)$ & $1.92(0.18-10.58)$ & \\
\hline Positive & $35(19.34 \%)$ & $3.51(1.12-19.29)$ & \\
\hline Missing & $6(3.31 \%)$ & & \\
\hline Differentiation (Edmonson) & & & 0.439 \\
\hline $\mathrm{I}+\mathrm{II}$ & $125(69.06 \%)$ & $2.14(0.22-10.18)$ & \\
\hline III + IV & $52(28.73 \%)$ & $3.85(0.22-18.82)$ & \\
\hline Missing & $4(2.21 \%)$ & & \\
\hline TNM stage & & & 0.046 \\
\hline $\mathrm{I}+\mathrm{II}$ & $122(67.40 \%)$ & $1.31(0.19-9.03)$ & \\
\hline III + IV & $54(29.83 \%)$ & $3.65(0.86-17.54)$ & \\
\hline Missing & $5(2.76 \%)$ & & \\
\hline
\end{tabular}

${ }^{a}$ Methylation level was displayed as median (interquartile range). ${ }^{b} \mathrm{p}$-value was calculated by Mann-Whitney $\mathrm{U}$ test and $\mathrm{p}<0.05$ was considered statistically significant (in bold).

\section{Discussion}

It is well known that aberrant methylation plays a pivotal role in hepatocarcinogenesis (7). TRIM58 methylation was first reported in HCC by methylation microarrays, and validated in only 10 paired primary tumor and adjacent liver tissues (13).
However, the authors did not perform any clinical associations with their data due to the limited samples. In this study, detailed TRIM58 methylation of $30 \mathrm{CpG}$ sites at a 343 bp CGI (CpG islands) region was initially analyzed by BGS in a pilot cohort of 30 paired HCC and adjacent non-tumor tissues. The result indicated that the TRIM58 methylation increased significantly 

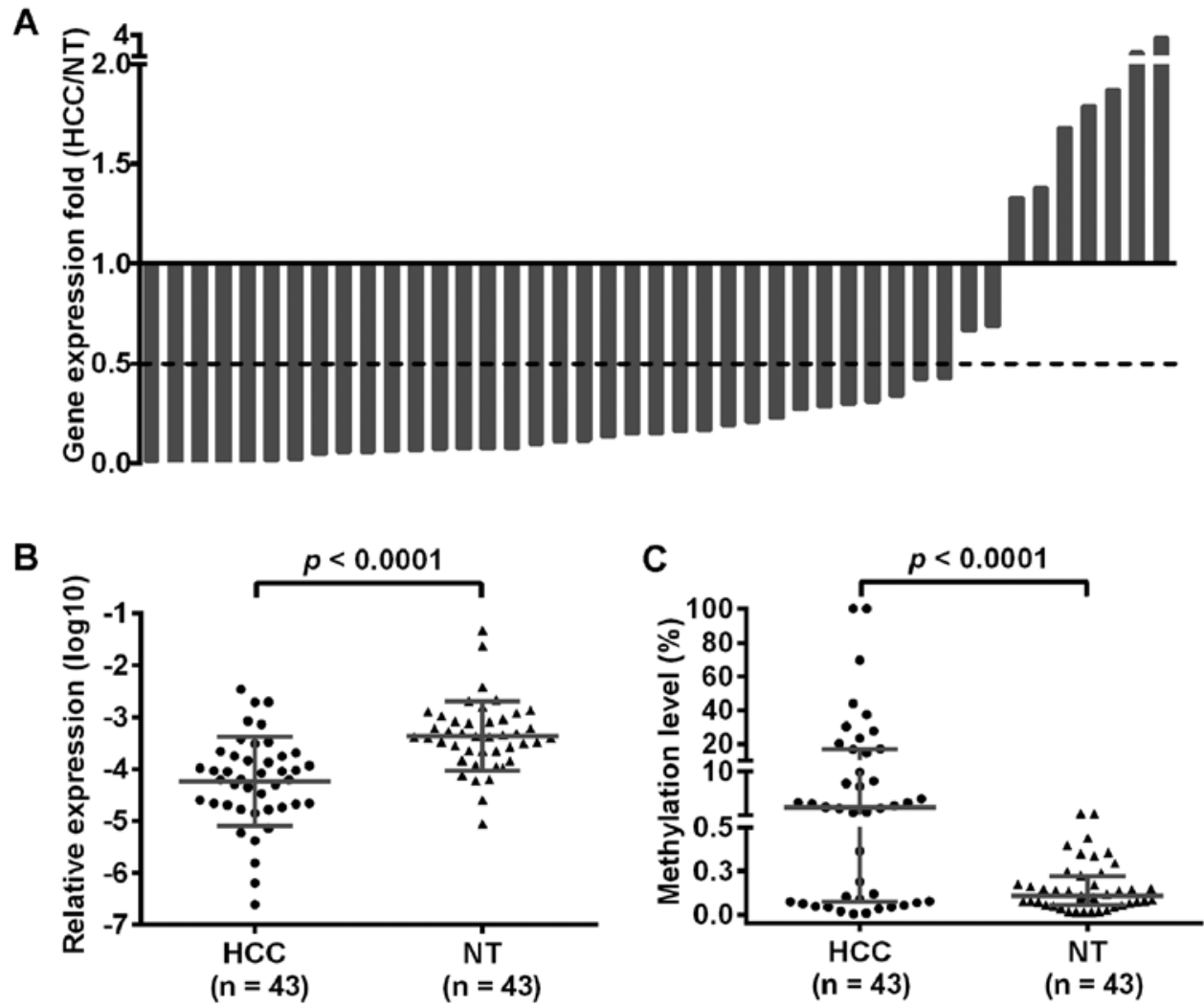

Figure 4. Correlations of TRIM58 expression and methylation in HCC and paired adjacent non-tumor tissues. (A) Quantitative real-time PCR analysis of the mRNA expression of TRIM58 in $43 \mathrm{HCC}$ and paired adjacent non-tumor tissues. TRIM58 downregulation was found in 79\% HCC tissues. (B) Comparison of TRIM58 expression in HCC and paired adjacent non-tumor tissues. The bars represented the mean \pm SD and the p-value was calculated by Paired t-test. (C) The methylation level of TRIM58 was analyzed in 43 pairs of HCC and adjacent non-tumor tissues. The bars represented the median value with interquartile range and the p-value was calculated by Wilcoxon matched pairs test. HCC, hepatocellular carcinoma tissues; NT, adjacent non-tumor tissues.

in HCC tissues compared with adjacent non-tumor tissues, and the methylation difference of $\mathrm{CpG}$ sites in cluster $1(-6 \sim+99)$ was more severe, which was in accordance with previous study from Tao et al (13). Furthermore, this observation was further validated in a larger cohort of 181 HCC tissues, 172 adjacent non-tumor tissues and 13 normal liver tissues using MSREqPCR. Additionally, the methylation of TRIM58 was inversely associated with its mRNA expression, and tended to correlate with tumor embolus, advanced TNM stage and unfavorable DFS after hepatectomy.

Both results of BGS and MSRE-qPCR showed an increased methylation level of TRIM58 in HCC tissues compared with non-tumor tissues and normal liver tissues. It further validated that gene specific hypermethylation was considered as a common epigenetic event in HCC (8). However, the significant difference between adjacent non-tumor tissues and normal liver tissues was not observed in this study, and hypermethylation of TRIM58 appeared in HCC tissues, but not in adjacent non-tumor tissues and normal liver tissues. The results indicated that hypermethylation of TRIM58 (>10\%) was one of the specific events associated with tumorigenesis rather than other benign pathological processes, such as inflammation and cirrhosis (18).

It is well documented that aberrant methylation showed the potential value of prognostic prediction for patients with HCC, which might assist in making therapeutic schedule after hepatectomy $(19,20)$. Consistently, we found that hypermethylation of TRIM58 was associated with worse DFS, although it was not an independent prognostic predictor for unfavourable DFS. This may be due to the relative short follow-up time and limited sample size. Nevertheless, it implied that TRIM58 hypermethylation was not conducive to the patient's DFS. In accordance with previous study, larger tumor size and high-grade differentiation (Edmondson) were independent unfavorable factors for DFS (21). In addition, a positive correlation between TRIM58 methylation and tumor embolus, advanced TNM stage, the characteristics of disease progression and unfavorable prognosis $(22,23)$, were observed in patients with HCC. These results suggested that TRIM58 methylation closely associated with aggressive biological behavior of HCC and might be served as a potential prognostic marker for HCC patients. Thus, hypermethylation of TRIM58 might play a crucial role in hepatocarcinogenesis, especially for the progressive and aggressive nature of this disease.

Gene specific hypermethylation frequently mediated transcriptional silencing of the associated gene and played an important role in tumorigenesis $(24,25)$. Numerous genes, such as SOCS1 (17), GSTPI (26), SFRPI (27), FOXD3 (28), SYK (29), $F A M 43 B$ (30), which are involved in tumor suppression, cell cycle regulation, apoptosis, and DNA repair have been shown to be suppressed by DNA hypermethylation in HCC. In our study TRIM58 mRNA expression was significantly reduced in HCC tissues comparing with adjacent non-tumor tissues, while the assessment of DNA methylation by MSRE-qPCR demonstrated that TRIM58 methylation was significantly increased in HCC tissues. Furthermore, $72 \mathrm{~h}$ treatment with demethylation agent 
(5-aza-dc) in HCC cell lines was able to upregulate TRIM58 mRNA expression (13). These results indicated that TRIM58 methylation was inversely correlated with the downregulation of its expression. Herein, we speculated that TRIM58 methylation might be involved in the mechanism of gene silencing and then participated in the development of HCC, although it needed further investigation.

Of note, the following limitations should be considered in the present study. Firstly, although we detected TRIM58 methylation in HCC tissues, adjacent non-tumor tissues and normal liver tissues, the methylation levels in tissues both from patients of chronic hepatitis and liver cirrhosis without HCC were unclear, which will assist in stating the continuous changes of methylation in hepatocarcinogenesis. Secondly, the mRNA expression analysis was conducted in a small sample size as our study was mainly based on FFPE specimens. Finally, our follow-up time was relatively short with limited sample size, resulting in low power to evaluate the association between TRIM58 methylation and DFS. Thus, further validation in a prospective and large-scale clinical study is needed.

In conclusion, our data showed that hypermethylation of TRIM58 is one of the specific events in HCC, and may contribute to the downregulation of its mRNA expression. Moreover, hypermethylation of TRIM58 tend to be associated with worse DFS after hepatectomy. However, the clinical application of TRIM58 needs to be further assessed with more samples with different pathophysiological processes and histological characteristics.

\section{Acknowledgements}

This research was supported by the National Basic Research Program of China (973 Program, 2012CB720605), the Science and Technology Research Plan of Wuhan City (2015060101010057).

\section{References}

1. Torre LA, Bray F, Siegel RL, Ferlay J, Lortet-Tieulent J and Jemal A: Global cancer statistics, 2012. CA Cancer J Clin 65 : 87-108, 2015.

2. El-Serag HB: Epidemiology of viral hepatitis and hepatocellular carcinoma. Gastroenterology 142: 1264-1273.e1, 2012.

3. Forner A, Llovet JM and Bruix J: Hepatocellular carcinoma. Lancet 379: 1245-1255, 2012.

4. Shibata $\mathrm{T}$ and Aburatani H: Exploration of liver cancer genomes. Nat Rev Gastroenterol Hepatol 11: 340-349, 2014.

5. Pogribny IP and Rusyn I: Role of epigenetic aberrations in the development and progression of human hepatocellular carcinoma. Cancer Lett 342: 223-230, 2014.

6. You JS and Jones PA: Cancer genetics and epigenetics: Two sides of the same coin? Cancer Cell 22: 9-20, 2012.

7. Um TH, Kim H, Oh BK, Kim MS, Kim KS, Jung G and Park YN: Aberrant $\mathrm{CpG}$ island hypermethylation in dysplastic nodules and early HCC of hepatitis B virus-related human multistep hepatocarcinogenesis. J Hepatol 54: 939-947, 2011.

8. Raggi C and Invernizzi P: Methylation and liver cancer. Clin Res Hepatol Gastroenterol 37: 564-571, 2013.

9. Mikeska T, Bock C, Do H and Dobrovic A: DNA methylation biomarkers in cancer: Progress towards clinical implementation. Expert Rev Mol Diagn 12: 473-487, 2012.

10. Sceusi EL, Loose DS and Wray CJ: Clinical implications of DNA methylation in hepatocellular carcinoma. HPB Oxf 13: 369-376, 2011.

11. Napolitano LM and Meroni G: TRIM family: Pleiotropy and diversification through homomultimer and heteromultimer formation. IUBMB Life 64: 64-71, 2012.
12. Thom CS, Traxler EA, Khandros E, Nickas JM, Zhou OY, Lazarus JE, Silva AP, Prabhu D, Yao Y, Aribeana C, et al: Trim58 degrades Dynein and regulates terminal erythropoiesis. Dev Cell 30: 688-700, 2014

13. Tao R, Li J, Xin J, Wu J, Guo J, Zhang L, Jiang L, Zhang W, Yang $\mathrm{Z}$ and Li L: Methylation profile of single hepatocytes derived from hepatitis B virus-related hepatocellular carcinoma. PLoS One 6: e19862, 2011.

14. Qiu X, Hu B, Huang Y, Deng Y, Wang X and Zheng F: Hypermethylation of ACP1, BMP4, and TSPYL5 in hepatocellular carcinoma and their potential clinical significance. Dig Dis Sci 61: 149-157, 2016.

15. Zhang X, Liu S, Shen C, Wu Y, Zhang L, Chen X and Lu F: DNA methylation consistency implicates the primary tumor cell origin of recurrent hepatocellular carcinoma. Epigenomics 7: 581-592, 2015.

16. Wang Y, Cheng J, Xu C, Liu S, Jiang S, Xu Q, Chen X, Zhuang H and Lu F: Quantitative methylation analysis reveals gender and age differences in p16INK4a hypermethylation in hepatitis B virus-related hepatocellular carcinoma. Liver Int 32: 420-428, 2012.

17. Zhang X, Wang J, Cheng J, Ding S, Li M, Sun S, Zhang L, Liu S, Chen X, Zhuang H, et al: An integrated analysis of SOCS1 downregulation in HBV infection-related hepatocellular carcinoma. J Viral Hepat 21: 264-271, 2014.

18. Gao XD, Qu JH, Chang XJ, Lu YY, Bai WL, Wang H, Xu ZX, An LJ, Wang CP, Zeng Z, et al: Hypomethylation of long interspersed nuclear element-1 promoter is associated with poor outcomes for curative resected hepatocellular carcinoma. Liver Int 34: 136-146, 2014.

19. Villanueva A, Portela A, Sayols S, Battiston C, Hoshida Y, Méndez-González J, Imbeaud S, Letouzé E, Hernandez-Gea V, Cornella H, et al; HEPTROMIC Consortium: DNA methylationbased prognosis and epidrivers in hepatocellular carcinoma. Hepatology 61: 1945-1956, 2015.

20. Mah WC, Thurnherr T, Chow PK, Chung AY, Ooi LL, Toh HC, Teh BT, Saunthararajah Y and Lee CG: Methylation profiles reveal distinct subgroup of hepatocellular carcinoma patients with poor prognosis. PLoS One 9: e104158, 2014.

21. Yan T, Zhao JJ, Bi XY, Zhao H, Huang Z, Li ZY, Zhou JG, Li Y, Li C, Cai JQ, et al: Prognosis of hepatocellular carcinoma: A study of 832 cases. Zhonghua Zhong Liu Za Zhi 35: 54-58, 2013 (In Chinese).

22. Tandon P and Garcia-Tsao G: Prognostic indicators in hepatocellular carcinoma: a systematic review of 72 studies. Liver Int 29: 502-510, 2009.

23. Lu W, Dong J, Huang Z, Guo D, Liu Y and Shi S: Comparison of four current staging systems for Chinese patients with hepatocellular carcinoma undergoing curative resection: Okuda, CLIP, TNM and CUPI. J Gastroenterol Hepatol 23: 1874-1878, 2008.

24. Fukushige $S$ and Horii A: DNA methylation in cancer: A gene silencing mechanism and the clinical potential of its biomarkers. Tohoku J Exp Med 229: 173-185, 2013.

25. Gan L, Chen S, Zhong J, Wang X, Lam EK, Liu X, Zhang J, Zhou T, Yu J, Si J, et al: ZIC1 is downregulated through promoter hypermethylation, and functions as a tumor suppressor gene in colorectal cancer. PLoS One 6: e16916, 2011.

26. Zhong S, Tang MW, Yeo W, Liu C, Lo YM and Johnson PJ: Silencing of GSTP1 gene by CpG island DNA hypermethylation in HBV-associated hepatocellular carcinomas. Clin Cancer Res 8: 1087-1092, 2002.

27. Kaur P, Mani S, Cros MP, Scoazec JY, Chemin I, Hainaut P and Herceg Z: Epigenetic silencing of sFRP1 activates the canonical Wnt pathway and contributes to increased cell growth and proliferation in hepatocellular carcinoma. Tumour Biol 33: 325-336, 2012.

28. He G, Hu S, Zhang D, Wu P, Zhu X, Xin S, Lu G, Ding Y and Liang L: Hypermethylation of FOXD3 suppresses cell proliferation, invasion and metastasis in hepatocellular carcinoma. Exp Mol Pathol 99: 374-382, 2015.

29. Shin SH, Lee KH, Kim BH, Lee S, Lee HS, Jang JJ and Kang GH: Downregulation of spleen tyrosine kinase in hepatocellular carcinoma by promoter $\mathrm{CpG}$ island hypermethylation and its potential role in carcinogenesis. Lab Invest 94: 1396-1405, 2014.

30. Xu X, Liu RF, Wan BB, Xing WM, Huang J and Han ZG: Expression of a novel gene FAM43B repressing cell proliferation is regulated by DNA methylation in hepatocellular carcinoma cell lines. Mol Cell Biochem 354: 11-20, 2011. 